\title{
Blob-Hole Structures as Non-Axisymmetric Equilibrium Solutions for Potential Vorticity Conserving Fluids*)
}

\author{
Yusuke KOSUGA $^{1,2)}$ and Patrick H. DIAMOND ${ }^{1,3)}$ \\ 1) WCI Center for Fusion Theory, NFRI, Daejeon, Korea \\ ${ }^{2)}$ IAS and RIAM, Kyushu University, Fukuoka 816-8580, Japan \\ ${ }^{3)}$ CMTFO and CASS, UCSD, La Jolla, CA, USA
}

(Received 7 December 2012 / Accepted 1 May 2013)

\begin{abstract}
We characterize blob-hole structures as equilibrium solutions for potential vorticity (PV) conserving systems. To demonstrate this, we consider equilibrium statistical mechanics of PV conserving fluids. We calculate partition function and free energy of the system, under the constraints that the energy and all the moments of PV are conserved. Equilibrium solutions are obtained by minimizing the free energy. As an example of analytical solutions from this approach, we consider solutions that conserve the energy and potential enstrophy. The connection of the obtained solutions to blob-hole structures is discussed.
\end{abstract}

(c) 2013 The Japan Society of Plasma Science and Nuclear Fusion Research

Keywords: turbulence, equilibrium, potential vorticity, vortex solution

DOI: $10.1585 /$ pfr.8.2403080

\section{Introduction}

Understanding tokamak turbulence is essential for the success of ITER and DEMO. An important element in tokamak turbulence is a blob or hole structure, which is observed in the tokamak edge [1-3]. In the tokamak edge, the steep gradient of the profile flattens and creates a pair of a blob and hole. Once generated, these structures propagate - blobs propagate down the gradient while holes propagate up the gradient. Relaxation process due to these structures is quite different from conventional relaxation process by drift wave turbulence [4]. Indeed, these structures are more efficient in tapping free energy than drift waves, as these structures can drive subcritical instability $[4,5]$. In other words, structures can drive instability even when linear drift waves are predicted to be stable. Thus, structures are an important element in understanding tokamak phenomenology.

In order to understand physical property of these structures, several research is on-going [6-9]. However, while previous studies revealed basic physics of blobs and holes, they did not address the role of potential vorticity (PV) [10] in understanding blob/hole phenomenology. In plasmas, $\mathrm{PV}$ is viewed as the total charge, as PV $q=n_{\mathrm{e}}-\rho_{\mathrm{s}}^{2} \nabla_{\perp}^{2} \phi$ for Hasegawa-Mima [11] or HasegawaWakatani [12] system. At the simplest level, PV is conserved along trajectory produced by $E \times B$ convection, $\mathrm{d} q / \mathrm{d} t=0$. The conservation of PV has significant impact on turbulence dynamics and relaxation process. For example, in PV conserving system, both the energy and the potential enstrophy $\int \mathrm{d} x \mathrm{~d} y q^{2}$ are conserved. Due to

author'se-mail: kosuga@riam.kyushu-u.ac.jp

*) This article is based on the presentation at the 22nd International Toki Conference (ITC22). this property, the dual cascade of energy and potential enstrophy can occur, and the inverse cascade of the energy can pump zonal flows at the large scale $[13,14]$. More recently, an exact momentum budget for drift wave turbulence and zonal flows was proved for such PV conserving system [15]. Given these impact of PV conservation on turbulence dynamics or relaxation, then this naturally raises a question regarding the consequence of PV conservation in blob-hole physics. For example, we might ask questions such as what is the impact of PV conservation on the generation of these structures.

In this paper, we discuss blobs and holes as equilibrium solutions in PV conserving systems. To do so, we consider equilibrium statistical mechanics for PV conserving fluids $[16,17]$. In this line of thought, we calculate partition function and free energy, under the constraints that energy and all the moments of PV is conserved. After deriving a general equation to determine equilibrium solutions under the constraints, we consider the special case that the energy and the second moment of PV, i.e. potential entrophy, are conserved. We obtain analytical solutions and discuss their connection to blob-hole structures.

The remainder of the paper is organized as follows. In section 2, we explain a basic model and construct statistical mechanics for the model. There, we calculate free energy $F[q]$ and partition function $\mathcal{Z}=\int \mathcal{D} q \exp (-F[q])$. We also derive an equation to determine equilibrium solutions, by minimizing the free energy. In section 3 , we consider a limited case that energy and potential enstrophy are conserved. Analytical solutions are presented. Section 4 is summary and discussion. 


\section{Statistical Mechanics of Potential Vorticity Conserving Fluids}

In this paper, we consider Hasegawa-Mima model for drift wave turbulence:

$$
\partial_{t}\left(1-\nabla_{\perp}^{2}\right) \phi+\hat{z} \times \nabla \phi \cdot \nabla\left(-\nabla_{\perp}^{2} \phi\right)+v_{*} \partial_{y} \phi=0 .
$$

Here, normalization is standard, i.e.

$$
\omega_{\mathrm{ci}} t \rightarrow t, \rho_{\mathrm{s}} \nabla \rightarrow \nabla, \frac{e \phi}{T_{\mathrm{e}}} \rightarrow \phi, \frac{\rho_{\mathrm{s}}}{L_{n}} \rightarrow v_{*},
$$

where $L_{n}^{-1}=-\partial_{x} \ln \langle n\rangle$ is the scale length in the mean density profile. $v_{*}$ measures the inhomogeneity in the system. A linearized solution of Eq. (1) is drift wave, whose dispersion relation is obtained as $\omega_{\boldsymbol{k}}=\omega_{* \mathrm{e}} /\left(1+\rho_{\mathrm{s}}^{2} k_{\perp}^{2}\right)$.

Eq. (1) has several conserved quantities. First, by multiplying Eq. (1) by $\phi$ and integrating over space, we obtain energy conservation:

$$
\frac{\partial}{\partial t} \frac{1}{2} \int \mathrm{d} x \mathrm{~d} y\left\{\phi^{2}+\left(\nabla_{\perp} \phi\right)^{2}\right\}=0 .
$$

Secondly, Eq. (1) conserves potential vorticity:

$$
\frac{\mathrm{d} q}{\mathrm{~d} t}=0
$$

where $q=\left(1-\nabla_{\perp}^{2}\right) \phi-v_{*} x$ is the potential vorticity for drift wave turbulence. It then follows that all the moments of $q$, $\Omega_{n} \equiv \int \mathrm{d} x \mathrm{~d} y q^{n}$, are conserved.

These conservation relations constraint behavior of the system. In particular, relaxation occurs while respecting these constraints. In order to account for relaxation process under these constraint, here we consider equilibrium statistical mechanics of PV conserving fluids. By using the method, we derive equilibrium solutions for Eq. (1) and show that these equilibrium solutions contain blob/hole structures.

At this point, it would be important to discuss how inviscid system approaches an equilibrium state irreversibly. This point may be clarified by recalling the argument by Lynden-Bell and Dupree on irreversible relaxation in collisionless Vlasov system [18,19]. The key to the discussion is the development of fine scales and the coarse-graining of these scales. In PV perspective, this argument is elaborated as follows. Namely, as relaxation proceeds, PV fluids are mixed by random turbulent advection. In this mixing process, each fluid element has the same PV that it was assigned in the initial position. Due to this property, after some mixing process, neighboring fluid elements may originate from distant locations and can have significantly different values of PV. This leads to fine scale variation of $\mathrm{PV}$, which can be arbitrarily fine in the inviscid system. However, such small scales eventually becomes so small that we cannot resolve by given physical resolutions. At this point, we introduce the coarse-graining to smooth out the variation over arbitrarily fine scales. It is this coarsegraining that introduces an effective collision in the system and thus allows irreversible evolution of the coarse-grained PV towards an equilibrium state.

Given there is an equilibrium state in PV conserving system, now we characterize the state by calculating the free energy $F[q]$ and the partition function $\mathcal{Z}=$ $\int \mathcal{D} q \exp (-F[q])$. In this approach $[16,17]$, the equilibrium state is characterized as the state with the minimal $F[q]$. Note this is analogous to the mean field solution in the phase transition model [20,21], where the mean field solution gives the minimal free energy and contributes to the partition function significantly via the stationary phase.

$F[q]$ for Eq. (1) is calculated as follows. To account for the conservation of energy and all the moments of $q$, $F[q]$ is given as

$$
F[q]=\frac{\beta}{2} \int \mathrm{d} x \mathrm{~d} y\left\{\phi^{2}+\left(\nabla_{\perp} \phi\right)^{2}\right\}+\sum_{n} \frac{\mu_{n}}{n} \int \mathrm{d} x \mathrm{~d} y q^{n} .
$$

Here $\beta$ and $\mu_{n}$ are Lagrange multipliers. To write Eq. (5) as a function of $q$, we introduce Green's function

$$
\left(1-\nabla_{\perp}^{2}\right) G\left(\boldsymbol{x}, \boldsymbol{x}^{\prime}\right)=\delta\left(\boldsymbol{x}-\boldsymbol{x}^{\prime}\right)
$$

This allows us to write $F[q]$ as

$$
\begin{aligned}
F[q]= & \frac{\beta}{2} \int \mathrm{d} x \mathrm{~d} y \mathrm{~d} x^{\prime} \mathrm{d} y^{\prime} q(\boldsymbol{x}) G\left(\boldsymbol{x}, \boldsymbol{x}^{\prime}\right) q\left(\boldsymbol{x}^{\prime}\right) \\
& -\beta \int \mathrm{d} x \mathrm{~d} y h(\boldsymbol{x}) q(\boldsymbol{x}) \\
& +\sum_{n} \frac{\mu_{n}}{n} \int \mathrm{d} x \mathrm{~d} y q^{n}+F_{0} .
\end{aligned}
$$

Here

$$
h(\boldsymbol{x}) \equiv-\int \mathrm{d} x^{\prime} \mathrm{d} y^{\prime} G\left(\boldsymbol{x}, \boldsymbol{x}^{\prime}\right) v_{*} x^{\prime},
$$

and

$$
F_{0} \equiv \frac{\beta}{2} \int \mathrm{d} x \mathrm{~d} y \int \mathrm{d} x^{\prime} \mathrm{d} y^{\prime} v_{*}^{2} x G\left(\boldsymbol{x}, \boldsymbol{x}^{\prime}\right) x^{\prime}
$$

$F_{0}$ does not depend on $q$ and gives a constant value; hence we neglect this term here after. Note that the free energy $F[q]$ has analogous form as the free energy for the phase transition model for magnetization [20]. In particular, if we only retain $\mu_{2}$ and $\mu_{4}$, the free energy has the same form as the free energy for the $\phi^{4}$ model with external field $h$ [21].

\section{Equilibrium Solutions for PV Con- serving Systems}

The equilibrium solutions are obtained by minimizing the free energy:

$$
\frac{\delta F[q]}{\delta q}=\beta \phi+\sum_{n} \mu_{n} q^{n-1}[\phi]=0 .
$$

By solving Eq. (10), we can obtain equilibrium solutions in Hasegawa-Mima system, that conserves the energy and all the moments of $q$. 


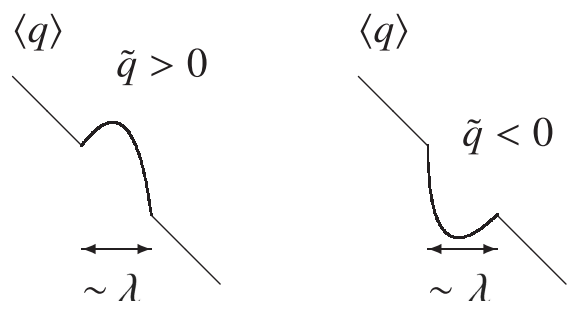

Fig. 1 A blob (a localized excess, $\tilde{q}>0$ ) and a hole (a localized depletion $\tilde{q}<0$ ) in the background mean PV $\langle q\rangle$. The structures are localized in the radial direction $x$, with the size $\lambda$.

Here, as an example of such solutions, we consider a limited case of $\mu_{n} \neq 0$ only for $n=2$. This corresponds to the case that the energy and potential enstrophy $\int \mathrm{d} x \mathrm{~d} y q^{2}$ are conserved. This case is akin to statistical mechanics developed by Kraichnan [13] or Hasegawa [14], for 2D or quasi-2D fluids with the energy and potential enstrophy conservation. In this limit, Eq. (10) reduces to

$$
\beta \phi+\mu_{2}\left\{\left(1-\nabla_{\perp}^{2}\right) \phi-v_{*} x\right\}=0 .
$$

Eq. (11) can be solved as

$$
\phi=\sum_{k} \phi_{k} \exp \left(i k_{y} y\right) \exp \left(-\frac{|x|}{\lambda_{k}}\right)+\frac{v_{*} x}{1+\beta / \mu_{2}},
$$

where $\lambda_{k}^{-2}=1+\beta / \mu_{2}+k_{y}^{2}$.

The equilibrium solution (Eq. (12)) describes solitary potential structures, as discussed in [22]. Solitary potential structures are related to PV blob/hole as follows. First, $\phi$ and $q$ are related as $q=-\left(\beta / \mu_{2}\right) \phi$ via Eq. (11). Note that $q=q[\phi]$ is indeed an equilibrium solution of HasegawaMima equation, since $E \times B$ flows cannot mix $q[\phi]$. The equilibrium $\mathrm{PV}$ is now $q=\langle q\rangle+\tilde{q}$, where

$$
\langle q\rangle=-\frac{\beta}{\mu_{2}} \phi_{0} \exp \left(-\frac{|x|}{\lambda_{0}}\right)-\frac{\beta}{\mu_{2}} \frac{v_{*} x}{1+\beta / \mu_{2}},
$$

is the zonally averaged background mean part and

$$
\tilde{q}=-\frac{\beta}{\mu_{2}} \sum_{k}^{\prime} \phi_{k} \exp \left(i k_{y} y\right) \exp \left(-\frac{|x|}{\lambda_{k}}\right),
$$

is the localized depletion/excess. Here the prime on the summation denotes the sum except for $k_{y}=0$. Typical configuration is depicted in Fig. 1. When $\tilde{q}>0, \tilde{q}$ gives a localized depletion, of the size $\sim \lambda \sim \rho_{\mathrm{s}}$. This corresponds to the PV blob. Similary, when $\tilde{q}<0, \tilde{q}$ gives a localized depletion, which can be viewed as the PV hole. Note that blobs $(\tilde{q}>0)$ and holes $(\tilde{q}<0)$ are equally probable in this formulation.

As a caveat, we note that the solution obtained here is only valid for non-axisymmetric case with $k_{z} \neq 0$, as PV $q=\left(1-\nabla_{\perp}^{2}\right) \phi-v_{*} x$ includes adiabatic electrons. However, in reality, plasmas support axisymmetric fluctuations with both $k_{z}=0$ and $k_{y}=0$, such as zonal flows, as well as nonaxisymmetric fluctuations. Then this naturally raises the question on how we reconcile zonal flows in this type of approach. To address this issue, here we note that there are at least two ways to include zonal flows in this story. The first way is to re-define PV for axisymmetric case and repeat the analysis for axisymmetric case. Note that the equilibrium statistical mechanics with PV conservation is applicable for axisymmetric zonal flows, since zonal modes also conserve $\mathrm{PV}, \mathrm{d} q / \mathrm{d} t=0$ with $q=\nabla_{\perp}^{2} \phi$. However, care must be taken in order to treat zonal flows in this approach. For example, axisymmetric solutions cannot be obtained by simply taking $k_{y}, k_{z} \rightarrow 0$ in Eq. (12). This is because Eq. (12) is obtained as non-axisymmetric solutions with adiabatic electrons with $k_{z} \neq 0, q=\left(1-\nabla_{\perp}^{2}\right) \phi-v_{*} x$. To treat axisymmetric solutions with $k_{z}=k_{y}=0$ in this approach, we must re-define some variables, as

$$
q=\nabla_{\perp}^{2} \phi,-\nabla_{\perp}^{2} G\left(\boldsymbol{x}, \boldsymbol{x}^{\prime}\right)=\delta\left(\boldsymbol{x}-\boldsymbol{x}^{\prime}\right), h \rightarrow 0 .
$$

With these redefinitions, we can now calculate the free energy with the energy and PV conservation. By minimizing the free energy we can obtain axisymmetric solutions. The second way to reconcile zonal flows is to consider the dynamics of non-axisymmetric solutions. Once formed, these non-axisymmetric solutions can grow by releasing the 'free energy' stored in the gradient. In this dynamical process, non-axisymmetric solutions (holes/blobs) can drive zonal flows by scattering polarization charge, which leads to zonal flow generation via the Taylor identity $\left\langle\tilde{v}_{x} \nabla_{\perp}^{2} \tilde{\phi}\right\rangle=\partial_{x}\left\langle\tilde{v}_{x} \tilde{v}_{y}\right\rangle$. Detailed analysis of the dynamical coupling of holes and zonal flows are given in literature, albeit the analysis is for phase space density holes [5].

\section{Conclusion and Discussion}

In this paper, we argued that blobs and holes are characterized as equilibrium solutions in potential vorticity (PV) conserving fluids. To demonstrate this, we developed equilibrium statistical mechanics, with the conservation of energy and all the moments of PV. We calculated the free energy with Lagrange multiplier. By minimizing the free energy, we obtained equilibrium solutions. As a specific example of such solutions, we discussed the case with the energy and potential enstrophy conserved. For this case, we obtained analytical solutions and discussed their relation to PV blobs and holes.

Theory presented here has relevant implication for the fusion community. Since tokamaks are strongly magnetized, potential vorticity conservation likely play a role in determining the behavior of the system. As demonstrated above, equilibrium solutions with PV conservation support blob/hole structures. Hence blobs and holes are likely present in a stationary state of strongly magnetized plasmas. This suggests that, while we tend to model tokamak turbulence as an ensemble of linear eigenmodes (such as drift waves), blob/hole structures cannot be neglected from transport analysis by tokamak turbulence. For example, such blobs or holes could play a role in the problem 


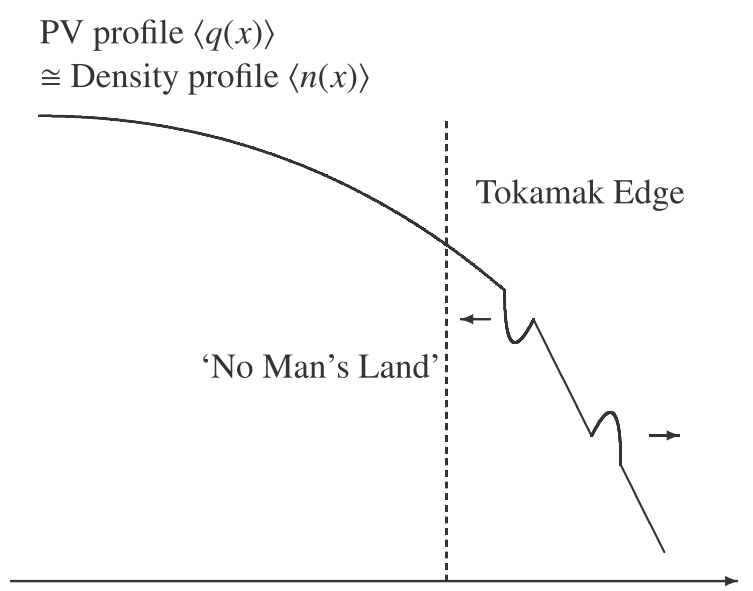

Fig. 2 PV holes and 'No Man's Land' [5]. The strong gradient at the edge can flatten and create a pair of a blob and hole. Once formed, a blob propagates down the gradient, while a hole propagates up the gradient. Such propagating hole structures can enter the edge-core coupling region ('No Man's Land') and can enhance fluctuations by extracting free energy in the local gradient, in addition to the linear instability driven by linear drift waves.

of the edge-core coupling region in tokamaks, so-called 'No Man's Land' (Fig. 2). In this region, recent validation study [23] found that turbulence level cannot be explained by simulations based on local models, which model turbulence by local instabilities driven by the local gradient. The hole structures discussed in this paper can contribute to the turbulence in NML non-locally. Once formed at the edge, these structures can propagate up the gradient and can extract free energy in these regions. Thus these structures may enhance turbulence level in the region, in addition to the linear instability driven by the local gradient. As a caveat, we note that the discussion presented here is heuristic, and hence a more detailed analysis is desirable to describe the dynamics of blob/hole structures. Such dynamics of blobs or holes involves turbulent transport of particles, which is non-zero only when there is a phase difference between density and potential fluctuations. Hence in order to describe the dynamics of blob/hole structures, we need a model that treats non-adiabatic electron response, such as Hasegawa-Wakatani model. The model for blob/hole dynamics based on Hasegawa-Wakatani turbulence is discussed elsewhere and we refer interested readers to the reference [24].

\section{Acknowledgement}

We thank T.S. Hahm, Ö.D. Gürcan, G. Dif-Pradalier, M. Lesur K. Itoh, S.-I. Itoh, J. Li, M. Sasaki, S. Sugita, and the participants in the 2009 and 2011 Festival de Theorie for stimulating discussions. This work was supported by CMTFO, the Ministry of Education, Science and Tech- nology of Korea via the WCI project 2009-001, U.S. Department of Energy Grants No. DE-FG02-04ER54738, and Grant-in-Aid for Scientific Research of JSPF of Japan (21224014).

[1] J.A. Boedo, D. Rudakov, R. Moyer, S. Krasheninnikov, D. Whyte, G. McKee, G. Tynan, M. Schaffer, P. Stangeby, P. West, S. Allen, T. Evans, R. Fonck, E. Hollmann, A. Lenard, A. Mahdavi, G. Porter, M. Tillack and G. Antar, Phys. Plasmas 8, 4826 (2001).

[2] J.A. Boedo, D.L. Rudakov, R.A. Moyer, G.R. McKee, R.J. Colchin, M.J. Schaffer, P.G. Stangeby, W.P. West, S.L. Allen, T.E. Evans, R.J. Fonck, E.M. Hollmann, S. Krasheninnikov, A.W. Leonard, W. Nevins, M.A. Mahdavi, G.D. Porter, G.R. Tynan, D.G. White and X. Xu, Phys. Plasmas 10, 1670 (2003).

[3] G.S. Xu, V. Naulin, W. Fundamenski, C. Hidalgo, J.A. Alonso, C. Silva, B. Goncalves, A.H. Nielson, J. Juul Rasmussen, S.I. Krasheninnikov, B.N. Wan, M. Stamp and JET EFDA Contributors, Nucl. Fusion 49, 092002 (2009).

[4] P.W. Terry, P.H. Diamond and T.S. Hahm, Phys. Fluids B 2, 2048 (1990).

[5] Y. Kosuga and P.H. Diamond, Phys. Plasmas 19, 072307 (2012).

[6] S.I. Krasheninnikov, Phys. Lett. A 283, 368 (2001).

[7] P. Beyer, S. Benkadda and G. Fuhr-Chaudier, Phys. Rev. Lett. 94, 105001 (2005).

[8] S. Sugita, M. Yagi, S.-I. Itoh and K. Itoh, J. Phys. Soc. Japan 79, 044502 (2010).

[9] S. Sugita, K. Itoh, S.-I Itoh, M. Yagi, G. Fuhr, P. Beyer and S. Benkadda, Plasma Phys. Control. Fusion 54, 125001 (2012).

[10] G.K. Vallis, Atmospheric and Oceanic Fluid Dynamics (Cambridge University Press, Cambridge, 2006).

[11] A. Hasegawa and K. Mima, Phys. Fluids 21, 87 (1978).

[12] A. Hasegawa and M. Wakatani, Phys. Rev. Lett. 50, 682 (1983).

[13] R.H. Kraichnan, J. Fluid Mech. 67, 155 (1975).

[14] A. Hasegawa, C.G. Maclennan and Y. Kodama, Phys. Fluids 22, 2122 (1979).

[15] P.H. Diamond, Ö.D. Gürcan, T.S. Hahm, K. Miki, Y. Kosuga and X. Garbet, Plasma Phys. Control. Fusion 50, 124018 (2008).

[16] J. Miller, Phys. Rev. Lett. 65, 2137 (1990).

[17] J. Miller, P.B. Weichman and M.C. Cross, Phys. Rev. A 45, 2328 (1992).

[18] D. Lynden-Bell, Mon. Not. R. Astr. Soc. 136, 101 (1967).

[19] T.H. Dupree, Phys. Fluids 26, 2460 (1983).

[20] L.P. Pitaevskii and E.M. Lifshitz, Physical Kinetics: Volume 10 (Course of Theoretical Physics) (Pergamon, Oxford, 1981).

[21] M. Kardar, Statistical Physics of Fields (Cambridge University Press, Cambridge, 2007).

[22] B.G. Hong, F. Romanelli and M. Ottaviani, Phys. Fluids B 3, 615 (1991).

[23] C. Holland, A.E. White, G.R. McKee, M.W. Shafer, J. Candy, R.E. Waltz, L. Schmitz and G.R. Tynan, Phys. Plasmas 16, 052301 (2009).

[24] Y. Kosuga, P.H. Diamond, L. Wang, Ö.D. Gürcan and T.S. Hahm, Nucl. Fusion 53, 043008 (2013). 